\title{
On Ordered Quasi-Gamma-Ideals of Regular Ordered Gamma-Semigroups
}

\author{
M. Y. Abbasi and Abul Basar \\ Department of Mathematics, Jamia Millia Islamia, New Delhi 110025, India \\ Correspondence should be addressed to Abul Basar; basar.jmi@gmail.com \\ Received 31 March 2013; Accepted 7 October 2013 \\ Academic Editor: Sorin Dascalescu
}

Copyright ( 2013 M. Y. Abbasi and A. Basar. This is an open access article distributed under the Creative Commons Attribution License, which permits unrestricted use, distribution, and reproduction in any medium, provided the original work is properly cited.

We introduce the notion of ordered quasi- $\Gamma$-ideals of regular ordered $\Gamma$-semigroups and study the basic properties of ordered quasi- $\Gamma$-ideals of ordered $\Gamma$-semigroups. We also characterize regular ordered $\Gamma$-semigroups in terms of their ordered quasi- $\Gamma$ ideals, ordered right $\Gamma$-ideals, and left $\Gamma$-ideals. Finally, we have shown that (i) a partially ordered $\Gamma$-semigroup $S$ is regular if and only if for every ordered bi- $\Gamma$-ideal $B$, every ordered $\Gamma$-ideal $I$, and every ordered quasi- $\Gamma$-ideal $Q$, we have $B \cap I \cap Q \subseteq(B \Gamma I \Gamma Q]$ and (ii) a partially ordered $\Gamma$-semigroup $S$ is regular if and only if for every ordered quasi- $\Gamma$-ideal $Q$, every ordered left $\Gamma$-ideal $L$, and every ordered right- $\Gamma$-ideal $R$, we have that $R \cap Q \cap L \subseteq(R \Gamma Q \Gamma L]$.

\section{Introduction}

Steinfeld [1-3] introduced the notion of a quasi-ideal for semigroups and rings. Since then, this notion has been the subject of great attention of many researchers and consequently a series of interesting results have been published by extending the notion of quasi-ideals to $\Gamma$-semigroups, ordered semigroups, ternary semigroups, semirings, $\Gamma$-semirings, regular rings, near-rings, and many other different algebraic structures [4-15].

It is a widely known fact that the notion of a one-sided ideal of rings and semigroups is a generalization of the notion of an ideal of rings and semigroups and the notion of a quasiideal of semigroups and rings is a generalization of a onesided ideal of semigroups and rings. In fact the concept of ordered semigroups and $\Gamma$-semigroups is a generalization of semigroups. Also the ordered $\Gamma$-semigroup is a generalization of $\Gamma$-semigroups. So the concept of ordered quasi-ideals of ordered semigroups is a generalization of the concept of quasi-ideals of semigroups. In the same way, the notion of an ordered quasi-ideal of ordered semigroups is a generalization of a one-sided ordered ideal of ordered semigroups. Due to these motivating facts, it is naturally significant to generalize the results of semigroups to $\Gamma$-semigroups and of $\Gamma$-semigroups to ordered $\Gamma$-semigroups.
In 1998, the concept of an ordered quasi-ideal in ordered semigroups was introduced by Kehayopulu [16]. He studied theory of ordered semigroups based on ordered ideals analogous to the theory of semigroups based on ideals. The concept of po- $\Gamma$-semigroup was introduced by Kwon and Lee in 1996 [17] and since then it has been studied by several authors [18-22]. Our purpose in this paper is to examine many important classical results of ordered quasi$\Gamma$-ideals in ordered $\Gamma$-semigroups and then to characterize the regular ordered $\Gamma$-semigroups through ordered quasi- $\Gamma$ ideals, ordered bi- $\Gamma$-ideals and ordered one-sided $\Gamma$-ideals.

\section{Preliminaries}

We note here some basic definitions and results that are relevant for our subsequent results.

Let $S$ and $\Gamma$ be two nonempty sets. Then $S$ is called a $\Gamma$ semigroup if $S$ satisfies $(a \gamma b) \mu c=a \gamma(b \mu c)$ for all $a, b, c \in S$ and $\gamma, \mu \in \Gamma$. A nonempty subset $N$ of a $\Gamma$-semigroup $S$ is called a sub- $\Gamma$-semigroup of $S$ if $a \alpha b \in N$ for all $a, b \in N$ and $\alpha \in \Gamma$. For any nonempty subsets $A, B$ of $S, A \Gamma B=\{a \alpha b$ : $a \in A, b \in B$ and $\alpha \in \Gamma\}$. We also denote $\{a\} \Gamma B, A \Gamma\{b\}$, and $\{a\} \Gamma\{b\}$, respectively, by $a \Gamma B, A \Gamma b$, and $a \Gamma b$. Many classical results of semigroups have been generalized and extended to $\Gamma$-semigroups [23-25]. By an ordered $\Gamma$-semigroup $S$ 
(also called po- $\Gamma$-semigroups), we mean an ordered set $(S, \leq)$, at the same time a $\Gamma$-semigroup satisfying the following conditions:

$$
a \leq b \Longrightarrow a \gamma c \leq b \gamma c, \quad c \gamma a \leq c \gamma b \forall a, b, c \in S, \gamma \in \Gamma \text {. }
$$

Throughout this paper, $S$ will stand for an ordered $\Gamma$ semigroup unless otherwise stated. An ordered $\Gamma$-semigroup $S$ is called regular if for each $s \in S$ and for each $\alpha, \beta \in \Gamma$ there exists $a \in S$ such that $s \leq s \alpha a \beta s$. Equivalent definitions of regular ordered $\Gamma$-semigroup are as follows: (i) $A \subseteq(A \Gamma S \Gamma A]$ for each $A \subseteq S$ and (ii) $s \in(s \Gamma S \Gamma s]$ for each $s \in S$. Let $(S ; \leq)$ be an ordered $\Gamma$-semigroup and $N$ a sub- $\Gamma$-semigroup of $S$; then $(N ; \leq)$ is an ordered $\Gamma$-semigroup. Let $A$ be a nonempty subset of $N$. Then similarly to [26], we write $(A]_{N}=\{n \in N: n \leq a$ for some $a \in A\}$ and $A \cup a=A \cup\{a\}$. We also write $(A]_{N}$ by simply $(A]$ if $N=S$ (see [27]). A nonempty subset $I$ of an ordered $\Gamma$-semigroup $S$ is called an ordered right- $\Gamma$-ideal (left- $\Gamma$-ideal) of $S$ if $I \Gamma S \subseteq I(S \Gamma I \subseteq I)$, and for any $x \in I$, $(x] \subseteq I$. $I$ is called an ordered $\Gamma$-ideal of $S$ if it is both a left and a right $\Gamma$-ideals of $S$. Also for any $s \in S$, we have that $(S \Gamma s]$ is an ordered left $\Gamma$-ideal of $S$ and $(s \Gamma S]$ is an ordered right $\Gamma$ ideal of $S$ [18]. A nonempty subset $Q$ of $S$ is called an ordered quasi- $\Gamma$-ideal of $S$ if (i) $(Q \Gamma S] \cap(S \Gamma Q] \subseteq Q$ and (ii) $(Q] \subseteq Q$. A sub- $\Gamma$-semigroup $B$ of an ordered $\Gamma$-semigroup $S$ is called an ordered bi- $\Gamma$-ideal of $S$ if $В Г S Г B \subseteq B$ and for any $x \in B$, $(x] \subseteq B$.

Let $X$ be a nonempty subset of $S$. Then the least right (left) ordered $\Gamma$-ideal of $S$ containing $X$ is given by $R(X)=(X \cup$ $X \Gamma S](L(X)=(S \Gamma X \cup X])$. If $X=\{s\}, s \in S$, we write $R\{s\}$ and $L\{s\}$, respectively, by $R(s)$ and $L(s)$, and $R(s)=(s \cup s \Gamma S]$, $L(s)=(S \Gamma s \cup s]$ and the ideal generated by $s \in S$ is given by $I(s)=(s \cup S \Gamma s \cup s \Gamma S \cup S \Gamma s \Gamma S]$. Also, the least quasi- $\Gamma$-ideal of $S$ containing $X$ is denoted by $Q(X)$. Moreover, we will need some notations as follows: (i) $N_{Q}=\{Q: Q \neq \emptyset$, where $Q \subseteq S$ and $(Q] \subseteq Q\}$, (ii) $R_{I}$ is a set of ordered right $\Gamma$-ideals of $S$, (iii) $L_{I}$ is a set of ordered left $\Gamma$-ideals of $S$, and (iv) $I_{T}$ is a two-sided $\Gamma$-ideal of $S$.

Now for any two elements $Q_{1}, Q_{2} \in N_{Q}$, we define an operation $*$ in $N_{Q}$ as follows:

$$
Q_{1} * \Gamma * Q_{2}=\left(Q_{1} \Gamma Q_{2}\right]
$$

Further, let $N$ be a sub- $\Gamma$-semigroup of $S$. Then we can easily observe here the following (see $[16,18,21,28-30])$ :

(i) $A \subseteq(A]_{N} \subseteq(A]=((A]]$ for $A \subseteq N$,

(ii) for $A \subseteq N$ and $B \subseteq N$, we have $(A \cup B]=(A] \cup(B]$,

(iii) for $A \subseteq N$ and $B \subseteq N$, we have $(A \cap B] \subseteq(A] \cap(B]$,

(iv) for $a$ and $b \in N$ with $a \leq b$, we have $(a \Gamma N] \subseteq(b \Gamma N]$ and $(N \Gamma a] \subseteq(N \Gamma b]$,

(v) $(A] \Gamma(B] \subseteq(A \Gamma B]$,

(vi) for every left (right, two-sided) ideal $L$ of $S,(L]=L$,

(vii) if $A$ and $B$ are ordered $\Gamma$-ideals of $S$, then $(A \Gamma B]$ and $A \cup B$ are also ideals of $S$,

(viii) for any $s \in S$, $(S \Gamma s \Gamma S]$ is an ideal of $S$.

\section{Ordered $\Gamma$-Semigroups and Ordered Quasi- $\Gamma$-Ideals}

In this section, we study some classical properties of the ordered $\Gamma$-semigroup $S$. We start with the following lemma.

Lemma 1. Let $S$ be an ordered $\Gamma$-semigroup. Then,

(i) $\left(N_{Q}, *, \subseteq\right)$ is an ordered $\Gamma$-semigroup;

(ii) $\left(L_{I}, *, \subseteq\right),\left(R_{I}, *, \subseteq\right)$, and $\left(I_{T}, *, \subseteq\right)$ are sub-Г-semigroups of $\left(N_{Q}, *, \subseteq\right)$.

Proof. (i) Suppose $P, Q, R \in N_{Q}$. Since $P \Gamma Q \in(P \Gamma Q]$, we obtain $((P \Gamma Q) \Gamma R] \subseteq((P \Gamma Q] \Gamma R]$. Next, we have $(P * \Gamma * Q) *$ $\Gamma * R=(P \Gamma Q \Gamma R]$ by using $(P * \Gamma * Q) * \Gamma * R=(P \Gamma Q] * \Gamma * R=$ $((P \Gamma Q] \Gamma R] \subseteq((P \Gamma Q) \Gamma R]=(P \Gamma Q \Gamma R]$. In a similar way, we can show that $P * \Gamma *(Q * \Gamma * R)=(P \Gamma Q \Gamma R]$ and therefore $(P * \Gamma * Q) * \Gamma * R=P * \Gamma *(Q * \Gamma * R)$. Hence $\left(N_{Q}, *\right)$ is a $\Gamma$-semigroup. Suppose $P \subseteq Q$. Then $P * \Gamma * R=(P \Gamma Q] \subseteq$ $(Q \Gamma R]=Q * \Gamma * R$ and $R * \Gamma * P=(R \Gamma P] \subseteq(R \Gamma Q]=R * \Gamma * Q$. Hence $\left(N_{Q}, *, \subseteq\right)$ is an ordered $\Gamma$-semigroup.

(ii) We have that $L_{I}, R_{I}$, and $I_{T}$ are nonempty subsets of $N_{Q}$. Suppose $L_{1}, L_{2} \in L_{I}$. Then, obviously, we have $\left(L_{1} * \Gamma *\right.$ $\left.L_{2}\right]=\left(\left(L_{1} \Gamma L_{2}\right]\right]=\left(L_{1} \Gamma L_{2}\right]$. Moreover, using

$$
\begin{aligned}
S \Gamma\left(L_{1} * \Gamma * L_{2}\right) & =S \Gamma\left(L_{1} \Gamma L_{2}\right] \\
& \subseteq\left(S \Gamma\left(L_{1} \Gamma L_{2}\right]\right] \\
& \subseteq\left(\left(S \Gamma L_{1}\right) \Gamma L_{2}\right] \\
& \subseteq\left(L_{1} \Gamma L_{2}\right] \\
& =L_{1} * \Gamma * L_{2},
\end{aligned}
$$

we infer that $L_{1} * \Gamma * L_{2}$ is a left $\Gamma$-ideal of $S$; that is, $L_{1} * \Gamma * L_{2} \epsilon$ $L_{I}$. Thus $\left(L_{I}, *, \subseteq\right)$ is a sub- $\Gamma$-semigroup of $\left(N_{Q}, *, \subseteq\right)$.

Dually, we can prove that $\left(R_{I}, *, \subseteq\right)$ is a sub- $\Gamma$-semigroup of $\left(N_{Q}, *, \subseteq\right)$. Since $I_{T}=L_{I} \cap R_{I}$, it follows that $\left(I_{T}, *, \subseteq\right)$ is a sub- $\Gamma$-semigroup of $\left(N_{Q}, *, \subseteq\right)$.

Let $Q_{I}=\{Q: Q$ is an ordered quasi- $\Gamma$-ideal of $S\}$. Then, obviously we have $L_{I} \cup R_{I} \subseteq Q_{I} \subseteq N_{Q}$. This implies that every one-sided $\Gamma$-ideal of an ordered $\Gamma$-semigroup is a quasi$\Gamma$-ideal of $S$. Thus the class of ordered quasi- $\Gamma$-ideals of $S$ is a generalization of the class of one-sided ordered $\Gamma$-ideals of $S$.

Lemma 2. Each ordered quasi- $\Gamma$-ideal $Q$ of an ordered $\Gamma$ semigroup $S$ is a sub-Г-semigroup of $S$.

Proof. Proof is straightforward. In fact, we have $Q \Gamma Q \subseteq Q \Gamma S \cap$ $S \Gamma Q \subseteq(Q \Gamma S] \cap(S \Gamma Q] \subseteq Q$

Lemma 3. For every ordered right $\Gamma$-ideal $R$ and an ordered left $\Gamma$-ideal $L$ of an ordered $\Gamma$-semigroup $S, R \cap L$ is an ordered quasi-Г-ideal of $S$.

Proof. As $R \Gamma L \subseteq S \Gamma L \subseteq L$ and $R \Gamma L \subseteq R \Gamma S \subseteq R$, we obtain $R \Gamma L \subseteq R \cap L$, so $R \cap L \neq \emptyset$. Now the fact that $R \cap L$ is an ordered quasi- $\Gamma$-ideal of $S$ follows from the following:

(i) $(R \cap L] \subseteq(R] \cap(L] \subseteq R \cap L$, 
(ii) $((R \cap L) \Gamma S] \cap(S \Gamma(R \cap L)] \subseteq(R \Gamma S] \cap(S \Gamma L] \subseteq(R] \cap(L] \subseteq$ $R \cap L$.

Lemma 4. Let $Q$ be an ordered quasi- $\Gamma$-ideal of $S$, then one obtains $Q=L(Q) \cap R(Q)=(S \Gamma Q \cup Q] \cap(Q \cup Q \Gamma S]$.

Proof. The following relation

$$
Q \subseteq(S \Gamma Q \cup Q] \cap(Q \cup Q \Gamma S] \text { is obvious. }
$$

Conversely, suppose $a \in(S \Gamma Q \cup Q] \cap(Q \cup Q \Gamma S]$. Then $a \leq b$ or $a \leq x \alpha u$ and $a \leq v \beta y$ for some $b, u, v \in Q, x, y \in S$, and $\alpha, \beta \in \Gamma$. As $Q$ is an ordered quasi- $\Gamma$-ideal of $S$, the former case implies that $a \in(Q] \subseteq Q$ and the latter case implies that $a \in(S \Gamma Q] \cap(Q \Gamma S] \subseteq Q$. Therefore $(S \Gamma Q \cup Q] \cap(Q \cup Q \Gamma S]=$ Q.

We recall here that if $X$ is a nonempty subset of an ordered $\Gamma$-semigroup $S$, then we write the least quasi-ideal of $S$ containing $X$ by $Q(X)$. If $X=\{a\}$, we write $Q(\{a\})$ by $Q(a)$.

Theorem 5. Suppose $S$ is an ordered $\Gamma$-semigroup. Then one has the following:

(i) for every $s \in S, Q(s)=L(s) \cap R(s)=(S \Gamma s \cup s] \cap(s \cup$ $s \Gamma S]$,

(ii) let $\emptyset \neq X \subseteq S, Q(X)=L(X) \cap R(X)=(S \Gamma X \cup X] \cap$ $(X \cup X \Gamma S]$.

Proof of (i). Suppose $s \in S$. Using Lemma 3, $L(s) \cap R(s)$ is a quasi- $\Gamma$-ideal of $S$ containing $s$; therefore $Q(s) \subseteq L(s) \cap R(s)$, and by Lemma 4 , we obtain

$$
\begin{aligned}
L(s) \cap R(s) & =(S \Gamma s \cup s] \cap(s \cup s \Gamma S] \\
& \subseteq(S \Gamma Q(s) \cup Q(s)] \cap(Q(s) \cup Q(s) \Gamma S] \\
& =Q(s) .
\end{aligned}
$$

Hence $Q(s)=L(s) \cap R(s)$.

Proof of (ii). Its proof can be given as (i).

The notion of a bi- $\Gamma$-ideal of $\Gamma$-semigroups is a generalization of the notion of a quasi- $\Gamma$-ideal of $\Gamma$-semigroups. Similarly, the class of ordered quasi- $\Gamma$-ideals of ordered $\Gamma$ semigroups is a particular case of the class of ordered bi- $\Gamma$ ideals of ordered $\Gamma$-semigroups. This is what we have shown in the following result.

Theorem 6. Suppose I is a two-sided ordered $\Gamma$-ideal of an ordered $\Gamma$-semigroup $S$ and $Q$ is a quasi- $\Gamma$-ideal of $I$; then $Q$ is an ordered bi-Г-ideal of $S$.

Proof. Since $Q$ is an ordered quasi- $\Gamma$-ideal of $I$ and $Q \subseteq I$, we obtain

$$
\begin{aligned}
\mathrm{Q} \Gamma Q & \subseteq \mathrm{Q} \Gamma S \Gamma I \\
& =\mathrm{Q} \Gamma(S \Gamma I) \subseteq Q \Gamma I \\
& \subseteq(Q \Gamma I] \subseteq(S \Gamma I] \subseteq(I] \subseteq I,
\end{aligned}
$$

$$
\begin{aligned}
& Q \Gamma S \Gamma Q \subseteq I \Gamma Q \Gamma S \\
&=(I \Gamma S) \Gamma Q \subseteq I \Gamma Q \subseteq(I \Gamma Q] \\
& \subseteq(I \Gamma S] \subseteq(I] \subseteq I,
\end{aligned}
$$

and $q \in(Q] \Rightarrow$ There exists $q_{1} \in Q \subseteq I$ such that $q \leq q_{1} \Rightarrow$ $q \in(I]=I$ and $q \in(Q] \Rightarrow q \in I \cap(Q]=(Q]_{I} \subseteq Q$.

Therefore,

$$
\begin{aligned}
\mathrm{Q} \Gamma S \Gamma \mathrm{Q} \subseteq(I \cap(I \Gamma Q]) \cap(I \cap(\mathrm{Q} \Gamma I]) \\
=(I \Gamma Q]_{I} \cap(\mathrm{Q} \Gamma]_{I} \subseteq \mathrm{Q}, \quad(\mathrm{Q}] \subseteq \mathrm{Q} .
\end{aligned}
$$

Hence applying these facts together with Lemma 2, we have shown that $Q$ is an ordered bi- $\Gamma$-ideal of $S$.

\section{Regular Ordered $\Gamma$-Semigroups and Ordered Quasi- $\Gamma$-Ideals}

In this section, we use the concept of ordered quasi- $\Gamma$-ideals to characterize regular ordered $\Gamma$-semigroups.

Lemma 7. Let $S$ be an ordered $\Gamma$-semigroup. Then the ordered sub-Г-semigroup of $\left(N_{Q}, *\right)$ generated by $\left(L_{I}, *\right)$ and $\left(R_{I}, *\right)$ is in the following form:

$$
\left\langle L_{I} \cup R_{I}\right\rangle=L_{I} \cup R_{I} \cup\left(R_{I} * \Gamma * L_{I}\right) .
$$

Proof. One can easily see that

$$
\begin{gathered}
\left\langle L_{I} \cup R_{I}\right\rangle=\left\{Y_{1} * \Gamma_{1} * Y_{2} * \cdots * Y_{n-1} * \Gamma_{n-1} * Y_{n} \mid Y_{j} \in L_{I}\right. \\
\text { or } \left.Y_{j} \in R_{I}, \quad j=1 \cdots n, n \in Z^{+}, \Gamma_{j} \in \Gamma\right\} .
\end{gathered}
$$

Suppose $Y_{j}, Y_{j+1} \in L_{I} \cup R_{I}$. Then the conditions that arise are as follows: (i) $Y_{j}, Y_{j+1} \in L_{I}$ : in this condition by Lemma 1, we obtain $Y_{j} * \Gamma * Y_{j+1} \in L_{I}$; (ii) $Y_{j}, Y_{j+1} \in R_{I}$ : in this condition, $Y_{j} * \Gamma * Y_{j+1} \in R_{I}$ by also Lemma 1; (iii) $Y_{j} \in L_{I}$, $Y_{j+1} \in R_{I}$ : in this condition, $Y_{j} * \Gamma * Y_{j+1}=\left(Y_{j} \Gamma Y_{j+1}\right]$ is an ordered $\Gamma$-ideal of $S$, so $Y_{j} * \Gamma * Y_{j+1} \in I_{T}=L_{I} \cap R_{I}$; (iv) $Y_{j} \in R_{I}$, $Y_{j+1} \in L_{I}$ : in this condition, $Y_{j} * \Gamma * Y_{j+1} \in R_{I} * \Gamma * L_{I}$ in $\left(N_{Q}, *\right)$. Therefore for any $Y_{1}, \ldots, Y_{n} \in L_{I} \cup R_{I}$, where $n \in Z^{+}$, using (i)-(iv), there arise three conditions as follows.

(i) $)^{\prime}$ If $Y_{1} \in L_{I}$, then $Y_{1} * \Gamma_{1} * Y_{2} * \cdots * Y_{n-1} * \Gamma_{n-1} * Y_{n} \in L_{I}$.

(ii) $^{\prime}$ If $Y_{n} \in R_{I}$, then $Y_{1} * \Gamma_{1} * Y_{2} * \cdots * Y_{n-1} * \Gamma_{n-1} * Y_{n} \in R_{I}$.

(iii) $^{\prime}$ If $Y_{1} \in R_{I}$ and $Y_{n} \in L_{I}$, where $n \geq 2$, then $Y_{1} * \Gamma_{1} *$ $Y_{2} * \cdots * Y_{n-1} * \Gamma_{n-1} * Y_{n} \in R_{I} * \Gamma * L_{I}$. Hence the lemma holds.

Theorem 8. Let $S$ be an ordered $\Gamma$-semigroup. Then the following assertions on $S$ are equivalent. 
(i) $S$ is a regular ordered $\Gamma$-semigroup.

(ii) For every ordered left $\Gamma$-ideal $L$ and every ordered right $\Gamma$-ideal $R$, one has

$$
(R \Gamma L]=R \cap L
$$

(iii) For every ordered right $\Gamma$-ideal $R$ and ordered left $\Gamma$ ideal $L$ of $S$,

(1) $(R \Gamma R]=R$,

(2) $(L \Gamma L]=L$,

(3) $(R \Gamma L]$ is an ordered quasi- $\Gamma$-ideal of $S$.

(iv) $\left(L_{I}, *\right)$ and $\left(R_{I}, *\right)$ are ordered idempotent $\Gamma$-semigroups and $\left(Q_{I}, *\right)$ is the sub-Г-semigroup of $\left(N_{Q}, *\right)$ generated by $\left(L_{I}, *\right)$ and $\left(R_{I}, *\right)$.

(v) $\left(Q_{I}, *\right)$ is a regular ordered sub- $\Gamma$-semigroup of the $\Gamma$ $\operatorname{semigroup}\left(N_{Q}, *\right)$.

(vi) Every ordered quasi- $\Gamma$-ideal $Q$ of $S$ is given by $Q=$ (QГSГQ].

(vii) $\left(Q_{I}, *, \subseteq\right)$ is a regular sub- $\Gamma$-semigroup of the ordered $\Gamma$-semigroup of $\left(N_{Q}, *, \subseteq\right)$.

Proof. (i) $\Rightarrow$ (ii) Suppose $R$ and $L$ are ordered right and left $\Gamma$-ideals of $S$, respectively; then we have

$$
(R \Gamma L] \subseteq R \cap L .
$$

Let $S$ be regular; we need to prove only that $R \cap L \subseteq(R \Gamma L]$. Suppose $a \in R \cap L$. Since $S$ is regular, we obtain $a \leq a \alpha x \beta a$ for some $x \in S$ and $\alpha, \beta \in \Gamma$, and so $a \in R$ and $x \alpha a \in L$; therefore $a \alpha x \beta a \in R \Gamma L$. Therefore $a \in(R \Gamma L]$, and thus $R \cap L \subseteq(R \Gamma L]$.

(ii) $\Rightarrow$ (iii) $(R \Gamma L]$ is an ordered quasi- $\Gamma$-ideal of $S$ that follows directly from Lemma 3 and the condition (ii). As the ordered two-sided $\Gamma$-ideal of $S$ is generated by $R=(R \cup S \Gamma R]$, the condition (ii) implies that

$$
\begin{aligned}
& R=R \cap(R \cup S \Gamma R]=(R \Gamma(R \cup S \Gamma R]) ; \\
& \text { therefore }(R \Gamma R] \subseteq(R \Gamma(R \cup S \Gamma R]]=R .
\end{aligned}
$$

Conversely, suppose $a \in(R \Gamma(R \cup S \Gamma R]]$. Then $a \leq r \alpha b$ for $r \in R$ and $b \in(R \cup S \Gamma R]$. From $b \in(R \cup S \Gamma R]$, we have $b \leq c$, where $c=r^{\prime} \in R$ or $c=s \alpha r^{\prime \prime}$ for some $s \in S$ and $r^{\prime \prime} \in R$. Therefore $a \leq r \alpha c=r \alpha r^{\prime} \in R \Gamma R$ or $a \leq r \alpha c=$ $r \alpha\left(s \beta r^{\prime \prime}\right)=(r \alpha s) \gamma r^{\prime \prime} \in R \Gamma R$ for $\alpha, \beta, \gamma \in \Gamma$; thus $a \in(R \Gamma R]$. Thus $R \subseteq(R \Gamma R]$, so that $(R \Gamma R]=R$. Similarly we can prove that $(L \Gamma L]=L$ dually.

(iii) $\Rightarrow$ (iv) The conditions (1), (2) in (iii) and Lemma 7 show that $\left(L_{I}, *\right)$ and $\left(R_{I}, *\right)$ are idempotent $\Gamma$-semigroups, respectively. Applying (iii) (3), we obtain $R_{I} * \Gamma * L_{I} \subseteq Q_{I}$; therefore $\left\langle L_{I} \cup R_{I}\right\rangle \subseteq Q_{I}$ in $\left(N_{Q}, *\right)$.

Conversely, suppose $Q \in Q_{I}$. Then $(Q \cup S \Gamma Q]$ is the ordered left $\Gamma$-ideal of $S$ generated by $Q$. The condition (iii) (2) implies that

$$
\begin{aligned}
Q \subseteq(Q \cup S \Gamma Q]= & ((Q \cup S \Gamma Q] \Gamma(Q \cup S \Gamma Q]] \\
\subseteq & (Q \Gamma Q \cup S \Gamma Q \Gamma Q \cup Q \Gamma S \Gamma Q \\
& \cup(S \Gamma Q) \Gamma(S \Gamma Q)] \subseteq(S \Gamma Q] .
\end{aligned}
$$

We can dually prove that $Q \subseteq(Q \Gamma S]$. Therefore using these facts and Lemma 4, it follows that

(a) $Q \subseteq(S \Gamma Q] \cap(Q \Gamma S] \subseteq(S \Gamma Q \cup Q] \cap(Q \cup Q \Gamma S]=Q$.

Therefore for $Q \in Q_{I}$, we have $Q=(S \Gamma Q] \cap(Q \Gamma S]$, and the condition (iii) (3) together with (a) implies that

(b) $\quad(R \Gamma L]=(S \Gamma(R \Gamma L]] \cap((R \Gamma L] \Gamma S]$.

Moreover, by the assertion (iii) (2), we have $S=(S \Gamma S]$ and

$$
\begin{aligned}
(S \Gamma Q] & =\left((S \Gamma Q]^{2}\right] \\
& =((S \Gamma Q] \Gamma(S \Gamma Q]]=((S \Gamma Q] \Gamma((S \Gamma S] \Gamma Q]] \\
& \subseteq(S \Gamma Q \Gamma S \Gamma S \Gamma Q] \subseteq(S \Gamma(Q \Gamma S] \Gamma(S \Gamma Q]]) \\
& \subseteq(S \Gamma((Q \Gamma S] \Gamma(S \Gamma Q]]] \subseteq(S \Gamma(Q \Gamma S \Gamma S \Gamma Q)] \\
& \subseteq(S \Gamma Q] .
\end{aligned}
$$

Therefore $(S \Gamma Q]=(S \Gamma((Q \Gamma S] \Gamma(S \Gamma Q]])$. Dually, we can prove that

$$
(Q \Gamma S]=(((Q \Gamma S] \Gamma(S \Gamma Q]] \Gamma S] .
$$

From these facts, (a) and (b), we obtain

$$
\begin{aligned}
\text { (c) } \quad Q & =(Q \Gamma S] \cap(S \Gamma Q] \\
& =(((Q \Gamma S] \Gamma(S \Gamma Q]] \Gamma S] \cap(S \Gamma((Q \Gamma S] \Gamma(S \Gamma Q]]) \\
& =((Q \Gamma S] \Gamma(S \Gamma Q]] \\
& =(Q \Gamma S] * \Gamma *(S \Gamma Q] \in R_{I} * \Gamma * L_{I} \\
& \subseteq\left\langle L_{I} \cup R_{I}\right\rangle
\end{aligned}
$$

by Lemma 7. Therefore $Q_{I} \subseteq\left\langle L_{I} \cup R_{I}\right\rangle$. Hence $Q_{I}=\left\langle L_{I} \cup R_{I}\right\rangle$ in $\left(N_{Q}, *\right)$.

(iv) $\Rightarrow$ (iii) It is a consequence of Lemma 7

(iii) $\Rightarrow$ (v) By (iii) $\Rightarrow$ (iv), we have (b) and (c). Suppose $Q_{1}, Q_{2}$ are two ordered quasi- $\Gamma$-ideals of $S$. Then $\left(S \Gamma\left(Q_{1} \Gamma Q_{2}\right] \cup\left(Q_{1} \Gamma Q_{2}\right]\right]$ is the least ordered left $\Gamma$-ideal of $S$ containing $\left(Q_{1} \Gamma Q_{2}\right]$. Then the condition (iii) (2) implies that

$$
\begin{aligned}
&\left(Q_{1} \Gamma Q_{2}\right] \subseteq\left(S \Gamma\left(Q_{1} \Gamma Q_{2}\right] \cup\left(Q_{1} \Gamma Q_{2}\right]\right] \\
&=\left(\left(S \Gamma\left(Q_{1} \Gamma Q_{2}\right] \cup\left(Q_{1} \Gamma Q_{2}\right]\right]^{2}\right] \\
& \subseteq\left(S \Gamma\left(Q_{1} \Gamma Q_{2}\right]\right]=\left((S \Gamma S] \Gamma\left(Q_{1} \Gamma Q_{2}\right]\right] \\
& \subseteq\left(S \Gamma\left(S \Gamma\left(Q_{1} \Gamma Q_{2}\right]\right]\right] .
\end{aligned}
$$


Dually one can prove that $\left(Q_{1} \Gamma Q_{2}\right] \subseteq\left(\left(Q_{1} \Gamma Q_{2}\right] \cup\right.$ $\left.\left(Q_{1} \Gamma Q_{2}\right] \Gamma S\right] \subseteq\left(\left(\left(Q_{1} \Gamma Q_{2}\right] \Gamma S\right] \Gamma S\right]$. These facts together with (b) show that

$$
\begin{aligned}
&\left(Q_{1} \Gamma Q_{2}\right] \subseteq\left(S \Gamma\left(Q_{1} \Gamma Q_{2}\right] \cup\left(Q_{1} \Gamma Q_{2}\right]\right] \\
& \cap\left(\left(Q_{1} \Gamma Q_{2}\right] \cup\left(Q_{1} \Gamma Q_{2}\right] \Gamma S\right] \\
& \subseteq\left(S \Gamma\left(S \Gamma\left(Q_{1} \Gamma Q_{2}\right]\right]\right] \\
& \cap\left(\left(\left(Q_{1} \Gamma Q_{2}\right] \Gamma S\right] \Gamma S\right] \\
&=\left(\left(\left(Q_{1} \Gamma Q_{2}\right] \Gamma S\right] \Gamma\left(S \Gamma\left(Q_{1} \Gamma Q_{2}\right]\right]\right] \\
& \subseteq\left(\left(Q_{1} \Gamma\left(Q_{2} \Gamma S \Gamma S\right) \Gamma Q_{1}\right) \Gamma Q_{2}\right] \subseteq\left(Q_{1} \Gamma Q_{2}\right] .
\end{aligned}
$$

By Theorem 5 (ii), $\left(Q_{1} \Gamma Q_{2}\right]=\left(S \Gamma\left(Q_{1} \Gamma Q_{2}\right] \cup\left(Q_{1} \Gamma Q_{2}\right]\right] \cap$ $\left(\left(Q_{1} \Gamma Q_{2}\right] \cup\left(Q_{1} \Gamma Q_{2}\right] \Gamma S\right]$ is an ordered quasi- $\Gamma$-ideal of $S$; therefore $Q_{1} * \Gamma * Q_{2} \in Q_{I}$. Hence $\left(Q_{I}, *\right)$ is a sub- $\Gamma$ semigroup of $\left(N_{Q}, *\right)$. For every $Q \in Q_{I}$, by (c), we obtain $Q=((Q \Gamma S] \Gamma(S \Gamma Q]] \subseteq(Q \Gamma S \Gamma S \Gamma Q] \subseteq(Q \Gamma S \Gamma Q] \subseteq Q$, and so $Q=(Q \Gamma S \Gamma Q]=Q * \Gamma * S * \Gamma * Q$, where $S \in Q_{I}$. Thus $\left(Q_{I}, *\right)$ is a regular sub $\Gamma$-semigroup of $\left(N_{Q}, *\right)$.

$(\mathrm{v}) \Rightarrow$ (vi) Suppose $Q$ is an ordered quasi- $\Gamma$-ideal of $S$. Applying the condition (iv), there is an ordered quasi- $\Gamma$-ideal $Q_{1}$ of $S$ so that, by Lemma 4,

$$
\begin{aligned}
Q & =Q * \Gamma * Q_{1} * \Gamma * Q=\left(Q \Gamma Q_{1} \Gamma Q\right] \\
& \subseteq(Q \Gamma S \Gamma Q] \subseteq(S \Gamma Q] \cap(Q \Gamma S] \\
& \subseteq(S \Gamma Q \cup Q] \cap(Q \cup Q \Gamma S]=Q,
\end{aligned}
$$

and therefore $Q=(Q \Gamma S \Gamma Q]$.

(vi) $\Rightarrow$ (vii) It is straightforward.

(vii) $\Rightarrow$ (i) For every $s \in S$, using Theorem $5, R(s) \cap L(s)$ is an ordered quasi- $\Gamma$-ideal of $S$ containing $s$. By (vii), there exists $Q \in Q_{S}$ so that

$$
\begin{aligned}
s \in R(s) \cap L(s) & \subseteq(R(s) \cap L(s)) * \Gamma * Q * \Gamma *(R(s) \cap L(s)) \\
& =((R(s) \cap L(s)) \Gamma Q \Gamma(R(s) \cap L(s))] \\
& \subseteq(R(s) \Gamma S \Gamma L(s)] \\
& =((s \cup s \Gamma S] \Gamma S \Gamma(S \Gamma s \cup s]] \subseteq(s \Gamma S \Gamma s] .
\end{aligned}
$$

Hence $S$ is a regular ordered $\Gamma$-semigroup.

Lemma 9. Every two-sided ordered $\Gamma$-ideal I of a regular ordered $\Gamma$-semigroup $S$ is a regular sub- $\Gamma$-semigroup of $S$.

Proof. Suppose $i \in I$. As $S$ is regular, there exists $s \in S$ so that, for $\alpha, \beta, \gamma, \delta \in \Gamma$, we have

$$
i \leq i \alpha s \beta i \leq i \alpha s \beta i \gamma s \delta i=i \alpha(s \beta i \gamma s) \delta i .
$$

As $s \alpha i \beta s \in S \Gamma I \Gamma S \subseteq I$, we observe that $i \in(i \Gamma I \Gamma i]_{I}$.

Theorem 10. Suppose $S$ is a regular ordered $\Gamma$-semigroup. Then the following statements are true. (i) Every ordered quasi- $\Gamma$-ideal of $S$ can be expressed as follows:

$$
Q=R \cap L=(R \Gamma L],
$$

where $R$ and $L$ are, respectively, the ordered right and left $\Gamma$-ideals of $S$ generated by $Q$.

(ii) Let $Q$ be an ordered quasi- $\Gamma$-ideal of S; then $(Q \Gamma Q]=$ (QГQГQ].

(iii) Every ordered bi- $\Gamma$-ideal of $S$ is an ordered quasi- $\Gamma$ ideal of $S$.

(iv) Every ordered bi- $\Gamma$-ideal of any ordered two sided- $\Gamma$ ideal of $S$ is a quasi- $\Gamma$-ideal of $S$.

(v) For every $L_{1}, L_{2} \in L_{I}$ and $R_{1}, R_{2} \in R_{I}$, one obtains

$$
\begin{aligned}
& L_{1} \cap L_{2} \subseteq\left(L_{1} \Gamma L_{2}\right], \\
& R_{1} \cap R_{2} \subseteq\left(R_{1} \Gamma R_{2}\right] .
\end{aligned}
$$

Proof. Because $S$ is a regular ordered $\Gamma$-semigroup, then by Lemma 4 and Theorem 8, the statement (i) is done. Since $(Q \Gamma Q \Gamma Q] \subseteq(Q \Gamma Q]$ is always true, we need to show that $(Q \Gamma Q] \subseteq(Q \Gamma Q \Gamma Q]$. We have that $(Q \Gamma Q]$ is also an ordered quasi- $\Gamma$-ideal of $S$ by Theorem 8 . Moreover we have the following equation:

$$
\begin{aligned}
(Q \Gamma Q] & =(Q \Gamma Q \Gamma S \Gamma Q \Gamma Q] \\
& =(Q \Gamma(Q \Gamma S \Gamma Q) \Gamma Q] \subseteq(Q \Gamma Q \Gamma Q] .
\end{aligned}
$$

Suppose $Q_{1}$ is an ordered bi- $\Gamma$-ideal of $S$. Then $\left(S \Gamma Q_{1}\right]$ is an ordered left $\Gamma$-ideal and $\left(Q_{1} \Gamma S\right]$ is an ordered right $\Gamma$-ideal of $S$. Applying Theorem 8, we obtain

$$
\begin{aligned}
\left(S \Gamma Q_{1}\right] \cap\left(Q_{1} \Gamma S\right] & =\left(\left(Q_{1} \Gamma S\right] \Gamma\left(S \Gamma Q_{1}\right]\right] \\
& \subseteq\left(Q_{1} \Gamma S \Gamma Q_{1}\right] \subseteq\left(Q_{1}\right] \subseteq Q_{1} .
\end{aligned}
$$

Therefore $Q_{1}$ is an ordered quasi- $\Gamma$-ideal of $S$.

Suppose $I$ is a two-sided ordered $\Gamma$-ideal of $S$ and $B$ is an ordered bi- $\Gamma$-ideal of $I$. By the relation (iii) and Lemma 9, $B$ is an ordered quasi- $\Gamma$-ideal of $I$; therefore using Theorem $6, B$ is an ordered bi- $\Gamma$-ideal of $S$. Also from the relation (iii) again, we obtain $B$ as an ordered quasi- $\Gamma$-ideal of $S$.

Lastly, suppose $L_{1}, L_{2} \in L_{I}$. Because $S$ is regular and $L_{1} \cap L_{2}$ is an ordered quasi- $\Gamma$-ideal of $S$, using Theorem 8 , we obtain

$$
\begin{aligned}
L_{1} \cap L_{2} & =\left(\left(L_{1} \cap L_{2}\right) \Gamma S \Gamma\left(L_{1} \cap L_{2}\right)\right] \\
& \subseteq\left(L_{1} \Gamma\left(S \Gamma L_{2}\right)\right] \subseteq\left(L_{1} \Gamma L_{2}\right] .
\end{aligned}
$$

Dually, we can prove that $R_{1} \cap R_{2} \subseteq\left(R_{1} \Gamma R_{2}\right]$ for all $R_{1}$, $R_{2} \in R_{I}$.

Theorem 11. A partially ordered $\Gamma$-semigroup $S$ is regular if and only if for every ordered $b i$ - $\Gamma$-ideal $B$, every ordered $\Gamma$-ideal $I$, and every ordered quasi- $\Gamma$-ideal $Q$, one has

$$
B \cap I \cap Q \subseteq(\text { ВГIГQ] } .
$$


Proof. Let $S$ be regular. Then for any $a \in B \cap I \cap Q$ there exists $s \in S$ such that

$$
\begin{aligned}
a & \leq a \alpha s \beta a \leq(a \alpha s \beta a) \gamma s \delta(a \alpha s \beta a) \\
& =(a \alpha s \beta a) \gamma(s \alpha a \beta s) \delta a \in(B \Gamma B) \Gamma(\text { SГIГS) ГQ } \\
& \subseteq B \Gamma I \Gamma Q .
\end{aligned}
$$

Hence $a \in(B \Gamma I \Gamma Q]$, where $\alpha, \beta, \gamma, \delta \in \Gamma$.

Conversely, let $B \cap I \cap Q \subseteq(B \Gamma I \Gamma Q]$ for every ordered bi$\Gamma$-ideal $B$, every ordered $\Gamma$-ideal $I$, and every ordered quasi$\Gamma$-ideal $Q$ of $S$. Suppose $s \in S$. Let $B(s)$ and $Q(s)$ be the ordered bi- $\Gamma$-ideal and ordered quasi- $\Gamma$-ideal of $S$ generated by $s$, respectively. So we have the following:

$$
\begin{aligned}
s \in B & (s) \cap I(s) \cap Q(s) \\
& \subseteq(B(s) \Gamma I(s) \Gamma Q(s)] \\
& \subseteq((s \cup s \Gamma S \Gamma s] \Gamma S \Gamma(s \cup(S \Gamma s \cap s \Gamma S)]] \\
& \subseteq((s \cup s \Gamma S \Gamma s] \Gamma S \Gamma(s \cup S \Gamma s]] \subseteq(s \Gamma S \Gamma s] .
\end{aligned}
$$

Hence $S$ is regular.

Next consider $R$ in place of $Q$ in Theorem 11 to obtain the following.

Corollary 12. An ordered $\Gamma$-semigroup $S$ is regular if and only if for every ordered bi- $\Gamma$-ideal $B$, every ordered $\Gamma$-ideal $I$, and every right $\Gamma$-ideal $R$ of $S$,

$$
B \cap I \cap R \subseteq(B Г I \Gamma R] .
$$

Theorem 13. A partially ordered $\Gamma$-semigroup $S$ is regular if and only iffor every ordered quasi- $\Gamma$-ideal $Q$, every ordered left $\Gamma$-ideal $L$, and every ordered right- $\Gamma$-ideal $R$, one has

$$
R \cap Q \cap L \subseteq(R \Gamma Q \Gamma L] .
$$

Proof. Let $S$ be regular; then for any $a \in R \cap Q \cap L$, there exists $s \in S$ such that $a \leq a \alpha s \beta a \leq(a \alpha s \beta a) \gamma s \delta(a \theta s \lambda a)=$ $(a \alpha s) \beta a \gamma(s \delta a \theta s \lambda a) \in(R \Gamma S) \Gamma Q \Gamma(S \Gamma L \Gamma S \Gamma L) \subseteq R \Gamma Q \Gamma L$, for $\alpha, \beta, \gamma, \theta, \delta, \lambda \in \Gamma$. Hence $a \in(R \Gamma Q \Gamma L]$.

Conversely, let

$$
R \cap Q \cap L \subseteq(R \Gamma Q \Gamma L],
$$

for every ordered right $\Gamma$-ideal $R$, every ordered quasi- $\Gamma$-ideal $Q$, and every ordered left $\Gamma$-ideal $L$ of $S$. Suppose $s \in S$. So we have

$$
\begin{aligned}
s \in R & (s) \cap Q(s) \cap L(s) \\
& \subseteq(R(s) \Gamma Q(s) \Gamma L(s)] \subseteq(R(s) \Gamma S \Gamma L(s)] \\
& \subseteq(R(s) \Gamma L(s)] \subseteq((s \cup s \Gamma S] \Gamma(s \cup S \Gamma s]] \\
& \subseteq((s \Gamma s \cup s \Gamma S \Gamma s]] .
\end{aligned}
$$

So for $\alpha, \beta, \gamma \in \Gamma, s \leq s \alpha s$ or $s \leq s \alpha x \beta s$ for some $x \in S$. If $s \leq s \alpha s$, then $s \leq s \alpha s \leq(s \alpha s) \beta(s \gamma s)=s \alpha(s \beta s)^{2} \gamma s \in s \Gamma S \Gamma s$. If $s \leq s \alpha x \beta s$ for some $x \in S$, then $s \in s \Gamma S \Gamma s$. So, finally we obtain $s \in(s \Gamma S \Gamma s]$. Hence $S$ is regular.
Corollary 14. If one considers an ordered left $\Gamma$-ideal L (or an ordered right $\Gamma$-ideal $R$ ) in place of the ordered quasi- $\Gamma$-ideal $Q$ in Theorem 13, one obtains

$$
L \cap R \subseteq(R \Gamma L] .
$$

\section{Acknowledgment}

The authors are grateful to the referee for the useful comments and valuable suggestions.

\section{References}

[1] O. Steinfeld, "On ideal-quotients and prime ideals," Acta Mathematica Academiae Scientiarum Hungaricae, vol. 4, pp. 289-298, 1953.

[2] O. Steinfeld, "Über die Quasiideale von Halbgruppen," Publicationes Mathematicae Debrecen, vol. 4, pp. 262-275, 1956.

[3] O. Steinfeld, Quasi-Ideals in Rings and Semigroups, vol. 10 of Disquisitiones Mathematicae Hungaricae, Akadémiai Kiadó, Budapest, Hungary, 1978.

[4] R. Chinram, "A note on Quasi-ideals in $\Gamma$-semirings," International Mathematical Forum, vol. 3, no. 25-28, pp. 1253-1259, 2008.

[5] R. Chinram, "On quasi gamma-ideals in $\Gamma$-semigroups," ScienceAsia, vol. 32, pp. 351-353, 2006.

[6] C. Dönges, “On Quasi-ideals of semirings,” International Journal of Mathematics and Mathematical Sciences, vol. 17, no. 1, pp. 47-58, 1994.

[7] A. H. Clifford, "Remarks on o-minimal Quasi-ideals in semigroups," Semigroup Forum, vol. 16, no. 2, pp. 183-196, 1978.

[8] P. Choosuwan and R. Chinram, "A study on Quasi-ideals in ternary semigroups," International Journal of Pure and Applied Mathematics, vol. 77, no. 5, pp. 39-647, 2012.

[9] V. N. Dixit and S. Dewan, "Minimal Quasi-ideals in ternary semigroup," Indian Journal of Pure and Applied Mathematics, vol. 28, no. 5, pp. 625-632, 1997.

[10] V. N. Dixit and S. Dewan, "A note on quasi and bi-ideals in ternary semigroups," International Journal of Mathematics and Mathematical Sciences, vol. 18, no. 3, pp. 501-508, 1995.

[11] K. Iseki, "Quasi-ideals in semirings without zero," Proceedings of the Japan Academy, vol. 34, pp. 79-81, 1958.

[12] R. D. Jagatap and Y. S. Pawar, "Quasi-ideals and minimal quasiideals in $\Gamma$-semirings," Novi Sad Journal of Mathematics, vol. 39, no. 2, pp. 79-87, 2009.

[13] N. Kehayopulu, S. Lajos, and G. Lepouras, "A note on biand Quasi-ideals of semigroups, ordered semigroups," Pure Mathematics and Applications, vol. 8, no. 1, pp. 75-81, 1997.

[14] S. Lajos, "On quasiideals of regular ring," Proceedings of the Japan Academy, vol. 38, pp. 210-211, 1962.

[15] I. Yakabe, "Quasi-ideals in near-rings," Mathematical Reports of College of General Education, Kyushu University, vol. 14, no. 1, pp. 41-46, 1983.

[16] N. Kehayopulu, "On completely regular ordered semigroups," Scientiae Mathematicae, vol. 1, no. 1, pp. 27-32, 1998.

[17] Y. I. Kwon and S. K. Lee, "Some special elements in ordered $\Gamma$-semigroups," Kyungpook Mathematical Journal, vol. 35, no. 3, pp. 679-685, 1996.

[18] A. Iampan and M. Siripitukdet, "On minimal and maximal ordered left ideals in PO-Г-semigroups," Thai Journal of Mathematics, vol. 2, no. 2, pp. 275-282, 2004. 
[19] A. Iampan, "Characterizing ordered bi-ideals in ordered $\Gamma$ semigroups," Iranian Journal of Mathematical Sciences \& Informatics, vol. 4, no. 1, pp. 17-25, 2009.

[20] A. Iampan, "Characterizing ordered Quasi-ideals of ordered $\Gamma$ semigroups," Kragujevac Journal of Mathematics, vol. 35, no. 1, pp. 13-23, 2011.

[21] Y. I. Kwon and S. K. Lee, "The weakly semi-prime ideals of po-Гsemigroups," Kangweon-Kyungki Mathematical Journal, vol. 5, no. 2, pp. 135-139, 1997.

[22] M. Siripitukdet and A. Iampan, "On the least (ordered) semilattice congruence in ordered $\Gamma$-semigroups," Thai Journal of Mathematics, vol. 4, no. 2, pp. 403-415, 2006.

[23] N. K. Saha, “On Г-semigroup II," Bulletin of the Calcutta Mathematical Society, vol. 79, no. 6, pp. 331-335, 1987.

[24] M. K. Sen and N. K. Saha, "On Г-semigroup I," Bulletin of the Calcutta Mathematical Society, vol. 78, no. 3, pp. 180-186, 1986.

[25] M. K. Sen, "On Г-semigroups," in Proceedings of the International Confernec on Algebra and It's Applications, pp. 301-308, Decker, New York, NY, USA, 1981.

[26] N. Kehayopulu, "On prime, weakly prime ideals in ordered semigroups," Semigroup Forum, vol. 44, no. 3, pp. 341-346, 1992.

[27] Y. Cao and X. Xinzhai, "Nil-extensions of simple posemigroups," Communications in Algebra, vol. 28, no. 5, pp. 2477-2496, 2000.

[28] N. Kehayopulu, "Note on Green's relations in ordered semigroups," Mathematica Japonica, vol. 36, no. 2, pp. 211-214, 1991.

[29] N. Kehayopulu, "On regular ordered semigroups," Mathematica Japonica, vol. 45, no. 3, pp. 549-553, 1997.

[30] N. Kehayopulu, "On weakly prime ideals of ordered semigroups,” Mathematica Japonica, vol. 35, no. 6, pp. 1051-1056, 1990. 


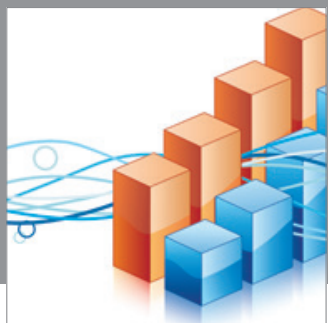

Advances in

Operations Research

mansans

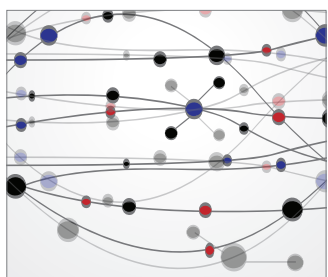

The Scientific World Journal
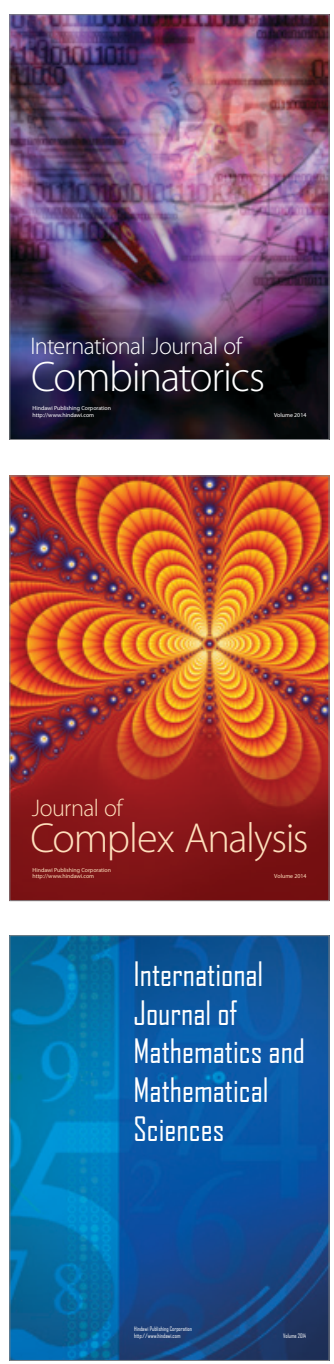
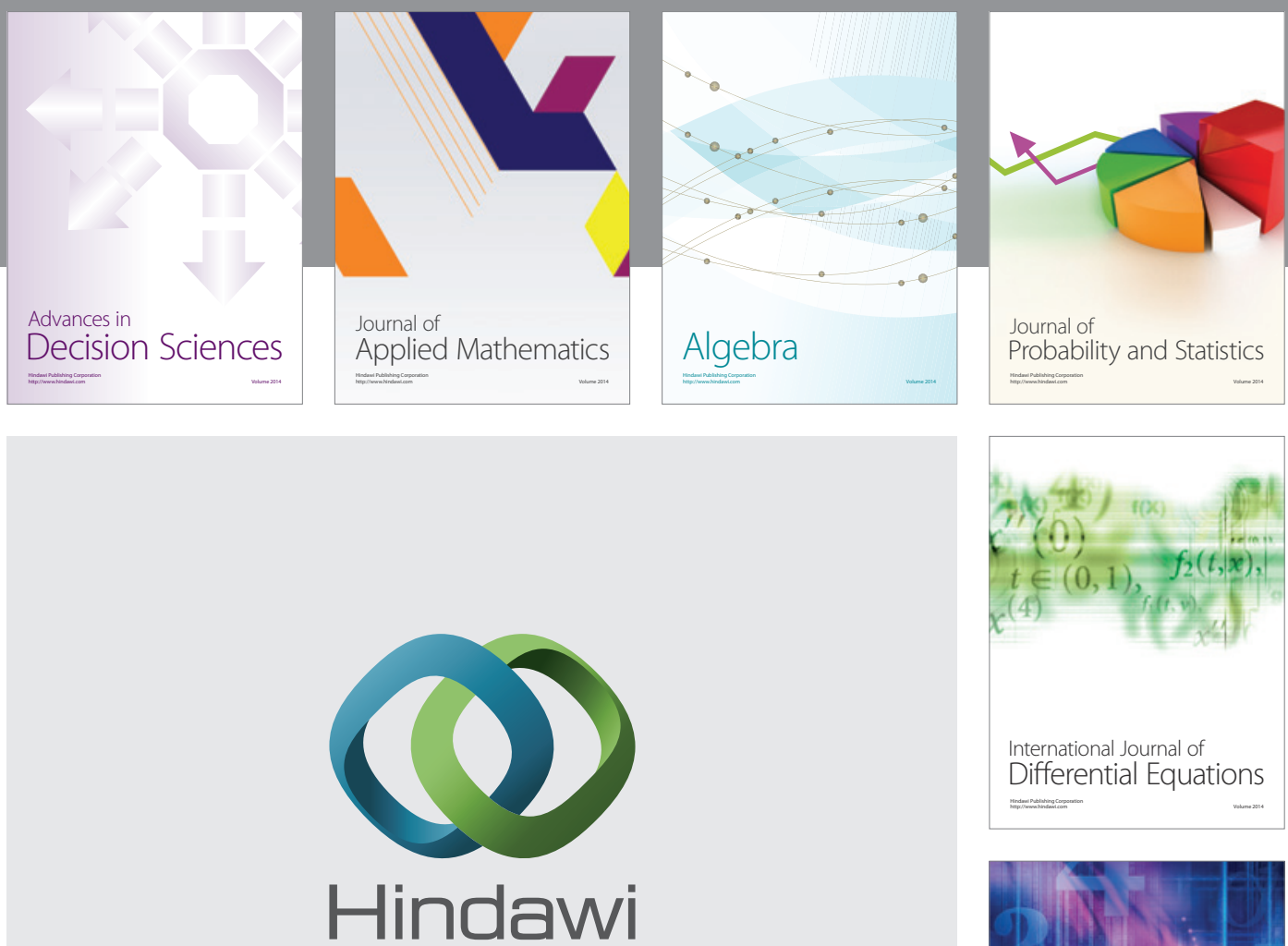

Submit your manuscripts at http://www.hindawi.com
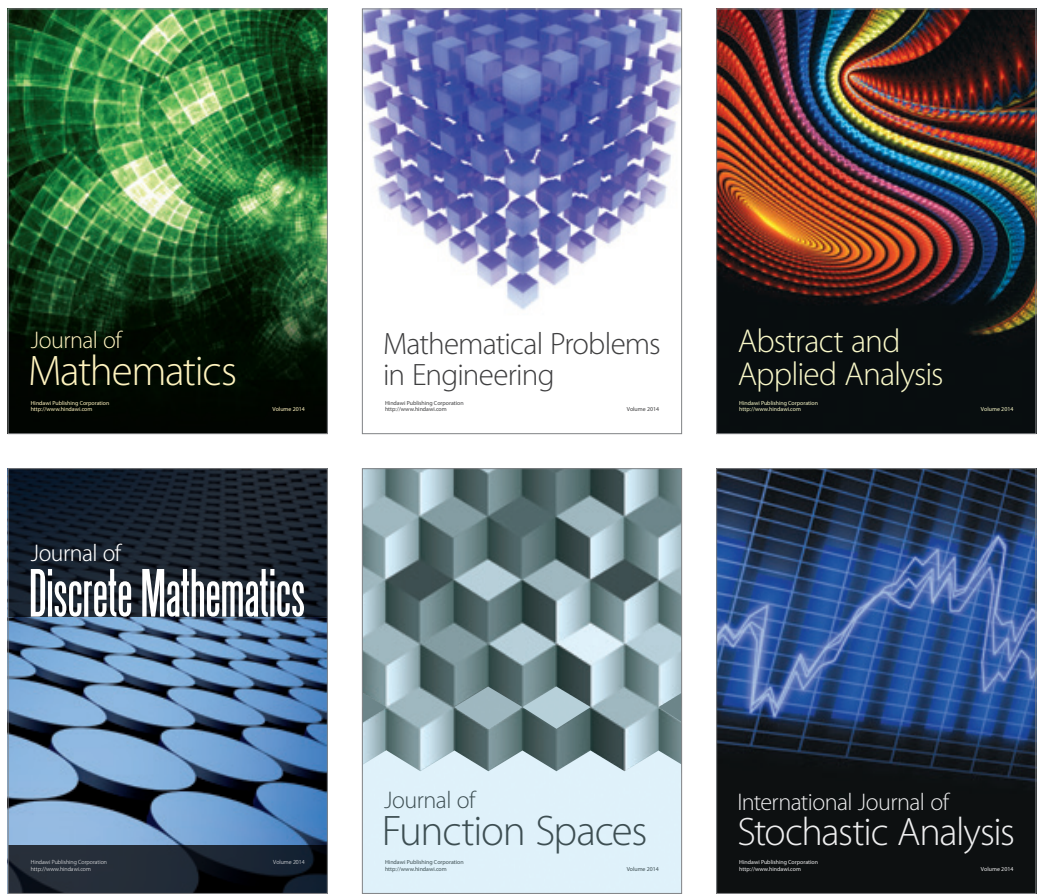

Journal of

Function Spaces

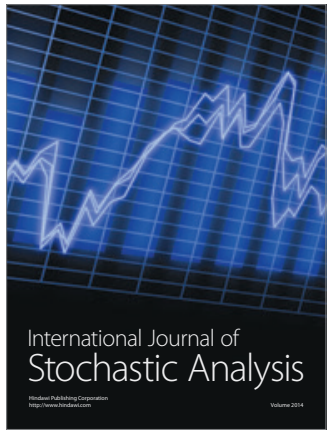

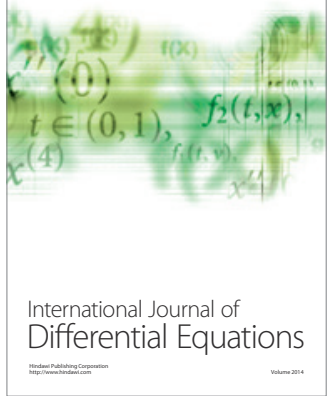
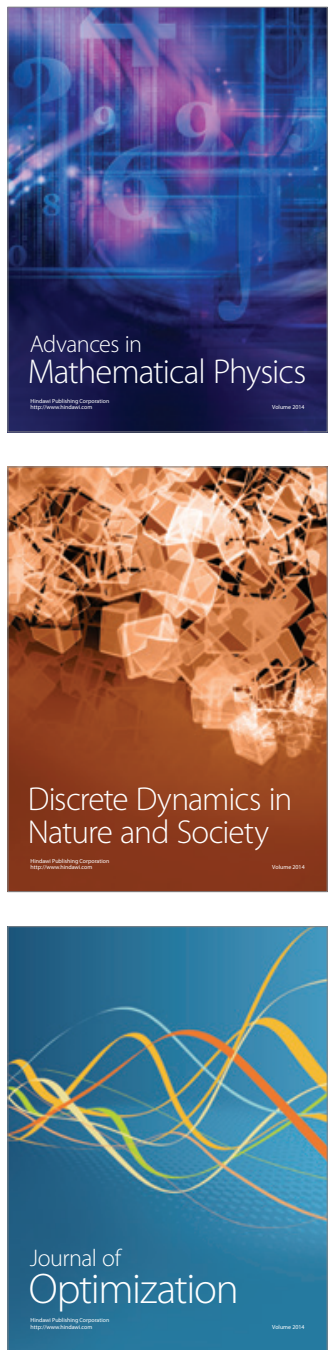